\title{
Aplicação de gabaritos na análise da dentadura decídua*
}

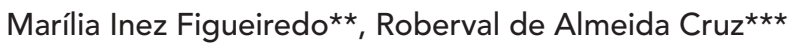

\section{Resumo}

Introdução: foram indicados, recentemente, gabaritos para analisar se a dentadura decídua encontra-se normal ou alterada. Objetivo: o presente estudo propôs aplicar os gabaritos desenvolvidos por Long em 59 modelos de dentaduras decíduas normais (grupo A) e 139 alteradas (grupo B), para avaliar sua eficiência clínica, à luz dos critérios clínicos conhecidos, que caracterizam uma oclusão normal e uma má oclusão. Metodologia: o grupo A, com 118 modelos de estudo de pacientes com oclusão normal, foi estratificado em 42 modelos de arcos superiores e 36 inferiores tipo I de Baume, e 17 modelos superiores e 23 inferiores de arcos tipo II. O grupo B, com 278 modelos de estudo com más oclusões diversas, foi composto por 78 arcos superiores e 75 inferiores tipo I, e 61 arcos superiores e 64 inferiores do tipo II. Examinou-se um total de 396 modelos. Resultados: os resultados do teste de Kappa entre as variáveis gabarito e critérios clínicos revelaram baixa concordância entre diagnóstico de normalidade e anormalidade da dentadura, independentemente do tipo de arco avaliado. Os gabaritos discordaram em 78\% dos casos diagnosticados clinicamente como normais e em 20\% dos diagnosticados com erros morfológicos. Conclusão: os gabaritos propostos não se aplicam à avaliação da dentadura decídua.

Palavras-chave: Dentadura decídua. Análise morfológica. Oclusão normal. Oclusão decídua. Má oclusão.

\section{INTRODUÇÃO E REVISÃO DE LITERATURA}

O diagnóstico de oclusão decídua normal e dos desvios morfológicos eventualmente presentes depende de critérios clínicos, vários deles baseados na avaliação da relação entre os arcos dentários. Assim, é importante observar a relação terminal dos segundos molares decíduos; o posicionamento dos caninos; os trespasses horizontal e vertical entre os incisivos; a presença ou ausência de espaçamentos, giroversões ou apinhamentos dentários ${ }^{6,15,17,21}$.

Entretanto, alguns desses fatores, como sobremordida e sobressaliência ocasionalmente aumen- tadas e presença de apinhamento, podem ser considerados normais para determinados estágios de desenvolvimento e não devem ser tratados ${ }^{24,26}$.

Por outro lado, a dentadura decídua pode ser influenciada por fatores intervenientes na morfologia dos arcos e, se forem instituídas abordagens multidisciplinares precoces, com a participação de outros profissionais de saúde (fonoaudiólogo, otorrinolaringologista, psicólogo, etc.), podem ser evitadas intervenções ortopédicas/ortodônticas, que só ocorreriam, dependendo do caso, na fase da dentadura mista $2,13,16,25$.

* Resumo da Dissertação de Mestrado (Pontifícia Universidade Católica - MG).

** Especialista em Ortodontia, Faculdade de Odontologia de Bauru/USP. Mestre em Odontopediatria, Pontifícia Universidade Católica /MG.

*** Livre-docente, coordenador dos programas de mestrado em Odontologia da Pontifícia Universidade Católica de Minas Gerais. 
As sobremordidas excessivas e más oclusões Classe II de Angle são acompanhadas e tratadas numa fase posterior do desenvolvimento oclusal, ao final da dentadura mista ${ }^{3,23,28}$. As sobressaliências aumentadas e mordidas abertas podem ser reduzidas com controle dos hábitos bucais, enquanto o tratamento dos aspectos oclusais pode ser postergado para um estágio mais desenvolvido da maturação dentária e do crescimento facial ${ }^{11,20,22,25}$. Portanto, qualquer que seja a situação clínica, há necessidade do estabelecimento de parâmetros que subsidiem o adequado diagnóstico, para ser planejado o correto tratamento, na época certa.

Do ponto de vista da dentadura decídua e seus aspectos de normalidade, foram descritas variadas formas de arcos dentários para caracterizá-la 1 1,7,21,30. Entretanto, por alguma razão, na maioria das vezes, qualquer que fosse o sistema adotado, a aplicação prática e sistematizada de parâmetros para a análise nunca foi disseminada na rotina clínica diária, embora sua adoção tenha sido recomendada ${ }^{15}$.

Há alguns anos, foi desenvolvido um sistema de gabaritos, gerados por computação eletrônica, para a determinação das posições dentárias ideais na dentadura decídua, dentro do intervalo considerado de normalidade ${ }^{17}$. Esse sistema foi aplicado em arcos dentários com más oclusões, tendo sido sugeridas soluções para as possíveis correções dos problemas detectados. Foi recomendado que, quando o ponto representativo do dente estiver alinhado ao contorno do arco elíptico, estará numa posição normal do arco. Em caso contrário, a avaliação orientará o diagnóstico ortodôntico e, conseqüentemente, o respectivo tratamento ${ }^{18}$. Os gabaritos foram sobrepostos em 30 pares de modelos de arcos dentários decíduos, de acordo com o tipo de arco caracterizado (I ou II de Baume), para verificar o posicionamento vestibulolingual de cada um dos dentes. Os resultados revelaram que $60 \%$ dos dentes superiores estavam vestibularizados em relação à posição ideal, enquanto, na mandíbula, 50\% dos dentes apresentaram bom posicionamento. Concluiu-se que os gabaritos eram de aplicação clínica simples, podendo ser muito úteis na determinação precoce de problemas na dentadura decídua ${ }^{29}$.

Considerando-se fisiologicamente a estabilidade da dentadura decídua, a necessidade do conhecimento de padrões de normalidade, a importância do diagnóstico precoce de más oclusões com etiologia multifatorial e a contribuição científica para comprovação de métodos pesquisados, foi proposta a verificação da aplicabilidade dos gabaritos de Long ${ }^{17}$ em modelos de dentaduras decíduas normais e alteradas, objetivando determinar sua acuidade e avaliar sua eficiência clínica, à luz dos critérios clínicos conhecidos que caracterizam a oclusão normal e a má oclusão.

\section{MATERIAL E MÉTODOS}

\section{Formação da amostra}

Foram avaliadas 1.500 crianças, para a seleção de 198, brasileiras, leucodermas, com idade entre 3 e 6 anos, de ambos os gêneros, com arcos dentários decíduos intactos e completos, sem cáries interproximais ou oclusais; distribuídas em dois grupos, caracterizados por modelos de estudo padrão, estratificados conforme o quadro 1, de acordo com o tipo de arco superior e inferior, tipo de arco de Baume $\mathrm{e}^{4}$ normalidade ou anormalidade da oclusão dentária; determinadas pelos critérios clínicos de $\operatorname{Long}^{17}$. A presença de qualquer dente permanente parcial ou totalmente irrompido e qualquer tratamento ortodôntico prévio representava critério diagnóstico de exclusão da criança na pesquisa.

\begin{tabular}{ccccc} 
& oclusão & \multicolumn{2}{c}{ arcos de Baume } & total de \\
& normal (grupo A) & 42 & 17 & 59 \\
crianças
\end{tabular}

Quadro 1 - Composição da amostra. 


\section{Critérios de diagnóstico para o grupo A - dentadura normal}

O grupo A foi composto por 59 crianças com dentaduras decíduas normais e a amostra dos 118 modelos obtidos foi definida de acordo com o quadro 1, segundo os critérios de Long $^{17}$, abaixo mencionados:

- espaçamento dos arcos dentários decíduos: espaços primatas ou generalizados (arco tipo I) ou sem espaçamentos (arco tipo II);

- relação terminal dos segundos molares decíduos em plano reto ou degrau mesial para a mandíbula;

- relação de caninos normal, com o vértice do canino superior no mesmo plano vertical da superfície distal do canino inferior, em oclusão cêntrica $^{12}$;

-relação de trespasse horizontal: relação de incisivos normal, positiva, com trespasse horizontal não excedendo $2 \mathrm{~mm}$ entre os incisivos superiores e inferiores ${ }^{12}$;

- relação de trespasse vertical: as bordas dos incisivos centrais inferiores contatando as palatinas dos incisivos centrais superiores, em oclusão cêntrica, não excedendo a metade de suas coroas clínicas $^{12}$.

\section{Critérios de diagnóstico para grupo B - dentaduras alteradas}

O grupo B foi composto por 139 crianças, com alterações morfológicas, devido a problemas diversos de oclusão, por exemplo: mordida aberta anterior, mordida cruzada posterior unilateral funcional, mordida cruzada posterior bilateral, mordida cruzada anterior, relação de caninos de Classe II de Angle, relação ântero-posterior de Classe III de Angle. Casos com relação de Classe I de caninos, com sobremordida profunda, sobressaliência aumentada e apinhamento dentário foram considerados má oclusão, segundo os padrões clínicos de normalidade ${ }^{17}$. Das 139 crianças, foram obtidos 278 modelos, estratificados de acordo com o quadro 1.

\section{Obtenção dos modelos de gesso}

Todos os modelos foram obtidos de forma padrão, com bases corretamente recortadas (Fig. 1, 2).

\section{Aplicação dos gabaritos}

- Foram marcados com lápis, nos modelos de gesso, pontos nas superfícies oclusais vestibulares

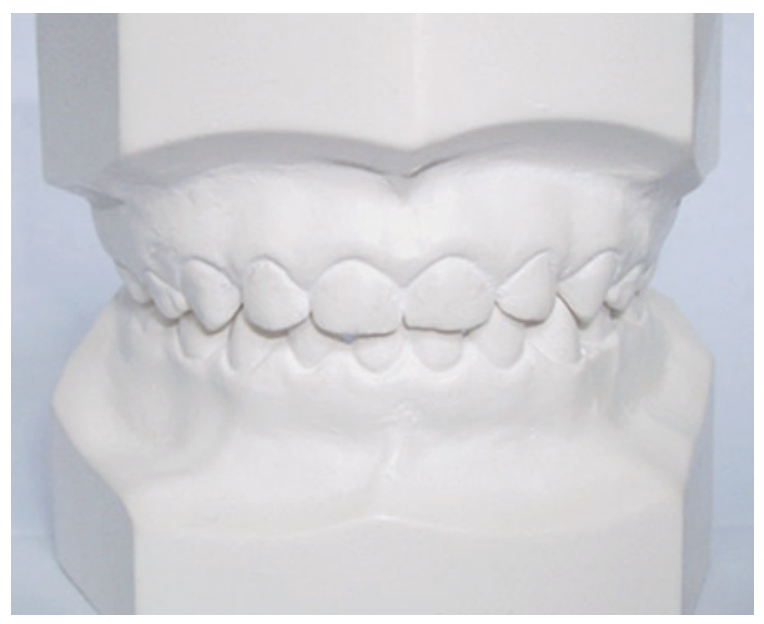

FIGURA 1 - Modelos de estudo - vista frontal.

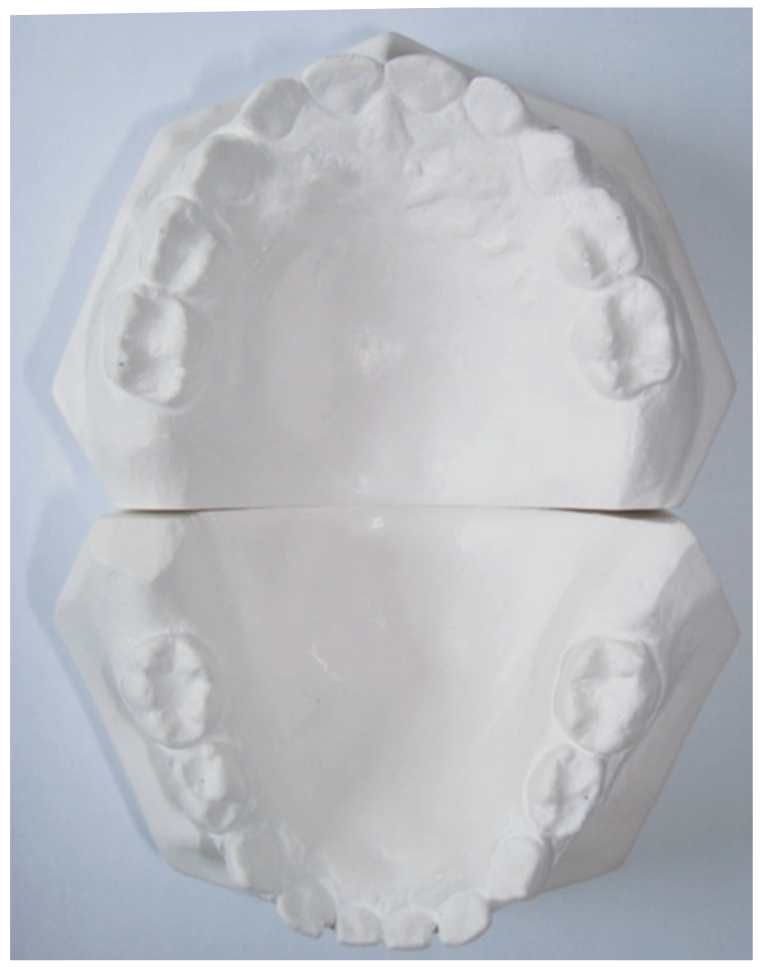

FIGURA 2 - Modelos de estudo - vistas oclusais (superior e inferior). 


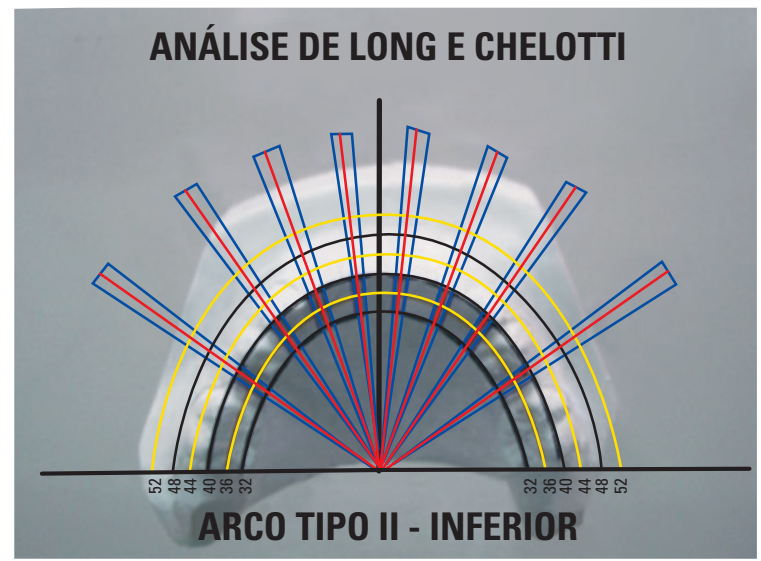

FIGURA 3 - Aplicação do gabarito ${ }^{18}$ sobre modelo de arco Tipo II inferior, normal.

dos dentes posteriores e incisais dos anteriores;

- selecionou-se o gabarito correspondente ao tipo de arco (I ou II de Baume), superior e/ou inferior;

- o gabarito foi aplicado sobre o modelo, coincidindo a reta base nas cúspides disto-vestibulares dos segundos molares decíduos, para estabelecer a distância molar (DM), tentando centralizar a perpendicular à reta base, de forma que passasse pelo ponto interincisivos;

- simultaneamente, a curva correspondente à forma do arco para essa DM deveria ter todos os pontos referentes aos dentes decíduos coincidindo sobre a curva selecionada (Fig. 3);

- se algum ponto representativo do dente não coincidisse com a curva de normalidade, seria considerado fora do padrão de normalidade (Fig. 4).

\section{Análise e interpretação dos dados}

O índice de Kappa forneceu o nível de concordância entre os dois métodos de diagnóstico da má oclusão: gabarito e critérios clínicos. Para avaliar a acuidade do gabarito no diagnóstico da má oclusão à luz dos critérios clínicos, foram aplicados os valores de: sensibilidade (S), especificidade (E), valor de predição positiva (VPP), valor de predição negativa (VPN), valores falso-positivos (FP) e falso-negativos (FN).

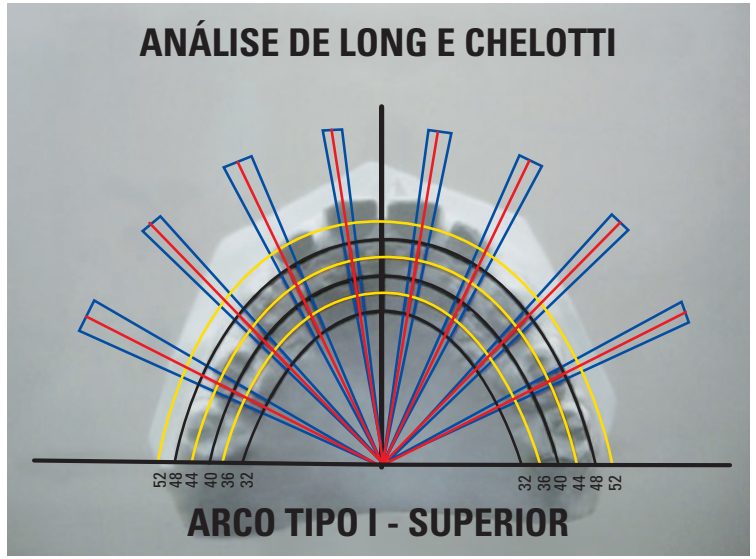

FIGURA 4 - Aplicação do gabarito ${ }^{18}$ sobre modelo de arco Tipo I superior, alterado.

\section{RESULTADOS}

Todos os resultados foram considerados significativos para uma probabilidade de significância inferior a 5\% (p < 0,05$)$. Há, portanto, pelo menos $95 \%$ de confiança nas conclusões apresentadas.

As tabelas 1 e 2 referem-se às medidas descritivas médias e individuais das distâncias molares encontradas em todos os modelos investigados.

Os resultados apresentados no quadro 2 e tabelas 3 a 6 mostram baixa concordância entre diagnóstico de normalidade e anormalidade da dentadura, segundo gabarito e critérios clínicos, independentemente do tipo de arco avaliado.

O quadro 3 mostra medidas de acuidade do gabarito à luz dos critérios clínicos, revelando que os resultados não são favoráveis para o gabarito.

\section{DISCUSSÃO}

Long ${ }^{17}$ avaliou 80 modelos de estudo de crianças com dentaduras decíduas normais para elaboração dos 4 gabaritos (2 superiores e 2 inferiores para arcos tipo I e II de Baume). Cada gabarito apresenta 6 elipses, com distâncias molares (DM) variando de $4 \mathrm{em} 4 \mathrm{~mm}(32,36,40,44,48,52)$, totalizando 24 elipses para representar o tamanho e a forma dos arcos decíduos (Fig. 5). Essas medidas parecem insuficientes pois, pelos resultados obtidos no presente estudo, verificou-se que 
Tabela 1 - Medidas descritivas da distância molar das crianças.

\begin{tabular}{cccccc} 
distância & \multicolumn{5}{c}{ medidas descritivas } \\
\cline { 2 - 6 } molar & mínimo & máximo & mediana & média & desvio \\
\hline superior & 40,0 & 52,0 & 46,0 & 45,9 & 2,2 \\
inferior & 36,0 & 47,0 & 42,0 & 41,6 & 2,2
\end{tabular}

Tabela 3 - Resultado do teste de concordância de Kappa para diagnóstico entre as variáveis “Gabarito e Critérios Clínicos" no arco Tipo I superior.

\begin{tabular}{ccccccc} 
& \multicolumn{4}{c}{ gabarito arco Tipo I superior } & \\
\cline { 2 - 5 } $\begin{array}{c}\text { critérios } \\
\text { clínicos }\end{array}$ & \multicolumn{2}{c}{ normal } & \multicolumn{2}{c}{ alterado } & total \\
& $\mathrm{n}$ & $\%$ & $\mathrm{n}$ & $\%$ & \\
normal & 8 & 19,0 & 34 & 81,0 & 42 \\
alterada & 10 & 12,8 & 68 & 87,2 & 78 \\
total & 18 & 15,0 & 102 & 85,0 & 120 \\
\multicolumn{5}{c}{ resultado do teste de Kappa } \\
estimativa $=0,072$ & & & $\mathrm{p}=0,362$ \\
\end{tabular}

Tabela 4 - Resultado do teste de concordância de Kappa para diagnóstico entre as variáveis "Gabarito e Critérios Clínicos" no arco Tipo II superior.

\begin{tabular}{|c|c|c|c|c|c|}
\hline \multirow{3}{*}{$\begin{array}{l}\text { critérios } \\
\text { clínicos }\end{array}$} & \multicolumn{4}{|c|}{ gabarito arco Tipo II superior } & \multirow{3}{*}{ total } \\
\hline & \multicolumn{2}{|c|}{ normal } & \multicolumn{2}{|c|}{ alterado } & \\
\hline & $\mathrm{n}$ & $\%$ & $n$ & $\%$ & \\
\hline normal & 3 & 17,6 & 14 & 82,4 & 17 \\
\hline alterada & 7 & 11,5 & 54 & 88,5 & 61 \\
\hline total & 10 & 12,8 & 68 & 87,2 & 78 \\
\hline \multicolumn{6}{|c|}{ resultado do teste de Карра } \\
\hline \multicolumn{3}{|c|}{ estimativa $=0,072$} & \multicolumn{3}{|c|}{$p=0,501$} \\
\hline
\end{tabular}

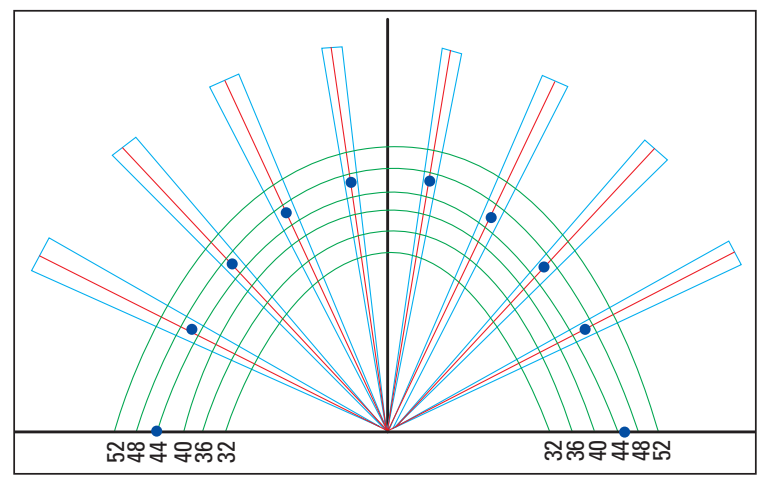

FIGURA 5 - Modelo do gabarito aplicado ${ }^{17}$.
Tabela 2 - Medidas descritivas das distâncias molares dos arcos dentários das crianças.

\begin{tabular}{|c|c|c|c|}
\hline \multirow{2}{*}{$\begin{array}{l}\text { distância } \\
\text { molar (mm) }\end{array}$} & \multicolumn{2}{|c|}{ oclusão } & \multirow{2}{*}{ total } \\
\hline & normal & alterada & \\
\hline \multicolumn{4}{|c|}{ superior } \\
\hline 40 & $1(1,7)$ & $1(0,7)$ & 2 \\
\hline 41 & $0(0,0)$ & $1(0,7)$ & 1 \\
\hline 42 & $2(3,4)$ & $5(3,6)$ & 7 \\
\hline 43 & $2(3,4)$ & $6(4,3)$ & 8 \\
\hline 44 & $14(23,7)$ & $33(23,7)$ & 47 \\
\hline 45 & $10(16,9)$ & $15(10,8)$ & 25 \\
\hline 46 & $11(18,6)$ & $21(15,1)$ & 32 \\
\hline 47 & $3(5,1)$ & $12(8,6)$ & 15 \\
\hline 48 & $13(22,0)$ & $32(23,0)$ & 45 \\
\hline 49 & $1(1,7)$ & $8(5,8)$ & 9 \\
\hline 50 & $1(1,7)$ & $2(1,4)$ & 3 \\
\hline 51 & $1(1,7)$ & $2(1,4)$ & 3 \\
\hline 52 & $0(0,0)$ & $1(0,7)$ & 1 \\
\hline total & $59(100,0)$ & $139(100,0)$ & 198 \\
\hline \multicolumn{4}{|c|}{ inferior } \\
\hline 36 & $1(1,7)$ & $0(0,0)$ & 1 \\
\hline 37 & $1(1,7)$ & $2(1,4)$ & 3 \\
\hline 38 & $3(5,1)$ & $13(9,4)$ & 16 \\
\hline 39 & $7(11,9)$ & $4(2,9)$ & 11 \\
\hline 40 & $20(33,9)$ & $29(20,9)$ & 49 \\
\hline 41 & $4(6,8)$ & $11(7,9)$ & 15 \\
\hline 42 & $12(20,3)$ & $20(14,4)$ & 32 \\
\hline 43 & $3(5,1)$ & $14(10,1)$ & 17 \\
\hline 44 & $7(11,9)$ & $36(25,9)$ & 43 \\
\hline 45 & $1(1,7)$ & $3(2,2)$ & 4 \\
\hline 46 & $0(0,0)$ & $6(4,3)$ & 6 \\
\hline 47 & $0(0,0)$ & $1(0,7)$ & 1 \\
\hline total & $59(100,0)$ & $139(100,0)$ & 198 \\
\hline
\end{tabular}

$\begin{array}{lcc}\text { arco de Baume } & \text { Kappa } & \text { p } \\ \text { maxila - Tipo I } & 0,072 & 0,362 \\ \text { maxila - Tipo II } & 0,072 & 0,501 \\ \text { mandíbula - Tipo I } & 0,055 & 0,549 \\ \text { mandíbula - Tipo II } & 0,170 & 0,072\end{array}$

Quadro 2 - Resumo do teste de Kappa com respectivos valores de significância. 
Tabela 5 - Resultado do teste de concordância de Kappa para diagnóstico entre as variáveis “Gabarito e Critérios Clínicos" no arco Tipo I inferior.

\begin{tabular}{|c|c|c|c|c|c|}
\hline \multirow{3}{*}{$\begin{array}{l}\text { critérios } \\
\text { clínicos }\end{array}$} & \multicolumn{4}{|c|}{ gabarito arco Tipo I inferior } & \multirow{3}{*}{ total } \\
\hline & \multicolumn{2}{|c|}{ normal } & \multicolumn{2}{|c|}{ alterado } & \\
\hline & n & $\%$ & $n$ & $\%$ & \\
\hline normal & 9 & 25,0 & 27 & 75,0 & 36 \\
\hline alterada & 15 & 20,0 & 60 & 80,0 & 75 \\
\hline total & 24 & 21,6 & 87 & 78,4 & 111 \\
\hline \multicolumn{6}{|c|}{ resultado do teste de Карра } \\
\hline \multicolumn{3}{|c|}{ estimativa $=0,055$} & \multicolumn{3}{|c|}{$p=0,549$} \\
\hline
\end{tabular}

\begin{tabular}{|c|c|c|c|}
\hline arco de Baume & níveis & teste & resultado \\
\hline \multirow{12}{*}{ maxila } & \multirow{6}{*}{ Tipo I } & sensibilidade & 87,2 \\
\hline & & especificidade & 19,0 \\
\hline & & valor predito positivo & 66,7 \\
\hline & & valor predito negativo & 44,4 \\
\hline & & falso-positivo & 33,3 \\
\hline & & falso-negativo & 55,6 \\
\hline & \multirow{6}{*}{ Tipo II } & sensibilidade & 88,5 \\
\hline & & especificidade & 17,6 \\
\hline & & valor predito positivo & 79,4 \\
\hline & & valor predito negativo & 30,0 \\
\hline & & falso-positivo & 20,6 \\
\hline & & falso-negativo & 70,0 \\
\hline \multirow{12}{*}{ mandíbula } & \multirow{6}{*}{ Tipo I } & sensibilidade & 80,0 \\
\hline & & especificidade & 25,0 \\
\hline & & valor predito positivo & 69,0 \\
\hline & & valor predito negativo & 37,5 \\
\hline & & falso-positivo & 31,0 \\
\hline & & falso-negativo & 62,5 \\
\hline & \multirow{6}{*}{ Tipo II } & sensibilidade & 92,2 \\
\hline & & especificidade & 21,7 \\
\hline & & valor predito positivo & 76,6 \\
\hline & & valor predito negativo & 50,0 \\
\hline & & falso-positivo & 23,4 \\
\hline & & falso-negativo & 50,0 \\
\hline
\end{tabular}

Quadro 3 - Resultados dos testes diagnósticos dos arcos superiores ou inferiores e tipos de arcos de Baume.
Tabela 6 - Resultado do teste de concordância de Kappa para diagnóstico entre as variáveis “Gabarito e Critérios Clínicos" no arco Tipo II inferior.

\begin{tabular}{|c|c|c|c|c|c|}
\hline \multirow{3}{*}{$\begin{array}{l}\text { critérios } \\
\text { clínicos }\end{array}$} & \multicolumn{4}{|c|}{ gabarito arco Tipo II inferior } & \multirow{3}{*}{ total } \\
\hline & \multicolumn{2}{|c|}{ normal } & \multicolumn{2}{|c|}{ alterado } & \\
\hline & $\mathrm{n}$ & $\%$ & $\mathrm{n}$ & $\%$ & \\
\hline normal & 5 & 21,7 & 18 & 78,3 & 23 \\
\hline alterada & 5 & 7,8 & 59 & 92,2 & 64 \\
\hline total & 10 & 11,5 & 77 & 88,5 & 87 \\
\hline \multicolumn{6}{|c|}{ resultado do teste de Kappa } \\
\hline \multicolumn{3}{|c|}{ estimativa $=0,170$} & \multicolumn{3}{|c|}{$p=0,072$} \\
\hline
\end{tabular}

existem vários modelos com $\mathrm{DM}$ intermediárias àquelas descritas (Tab. 2). Poderia ser sugerida a colocação de outras curvas intermediárias entre essas medidas; talvez, a confecção de gabaritos medidos de 1 em $1 \mathrm{~mm}$, com o objetivo de determinar exatamente a posição do dente em seu alvéolo. No entanto, ainda não existe qualquer aparelho ortodôntico, ortopédico ou removível que possa colocar os dentes milimetricamente em suas posições intra-alveolares, tanto no sentido vestibulolingual quanto mesiodistal. A autora da pesquisa consultada ${ }^{17}$ concorda que arcos dentários devem possuir dentes alinhados e nivelados em suas posições intra-alveolares, sem giroversões e sem inclinações vestibulares ou linguais, na fase de dentadura decídua, estágio curto e transitório, que, segundo Silva Filho et al. ${ }^{26}$, não deve ser analisada somente em seu aspecto intra-arco.

Os dados desta pesquisa revelaram a forma elíptica dos arcos decíduos em todos os 396 modelos estudados (59 crianças com normalidade de oclusão e 139 crianças com más oclusões diversas), representando variabilidade de arcos dentários suficiente para avaliar a aplicabilidade dos gabaritos testados, segundo análises estatísticas preliminares.

Silva Filho et al. ${ }^{27}$ comentaram também que a análise isolada do modelo superior é importante quando nos mostra que a forma é triangular e não parabólica, denunciando atresia maxilar, que pode 
levar a mordidas cruzadas.

O primeiro parâmetro sugerido para posicionar o gabarito foi a reta correspondente à distância molar medida. Gabaritos desenvolvidos para a dentadura permanente, como os de BeGole e Lyew $^{5}$ e Interlandi ${ }^{14}$, sugeriram a referência da curvatura anterior para determinar a forma do arco dentário humano. O comprimento do arco mostrou-se encurtado, pois os gabaritos ficaram aquém das curvaturas anteriores, evidenciando, em muitos casos, a vestibularização dos dentes anteriores, conforme também determinado por Zannet et al. ${ }^{29}$ Isso justifica o grande número, nesse estudo, de arcos dentários diagnosticados como normais pelos critérios clínicos e como alterados pelos gabaritos de Long. Os achados de $\mathrm{Carrea}^{7}$ mostraram também limitações em seus diagramas, por definir em 28, 30, 32 e 34mm as medidas de cada lado do triângulo equiilátero.

Depois de posicionada a reta referente à DM, Long $^{17}$ preconizou coincidir a perpendicular referente ao ponto interincisivos e, a partir daí, todos os pontos do gabarito deveriam esconder os pontos marcados nos dentes, que deveriam estar alinhados e nivelados para o diagnóstico de dentes decíduos bem posicionados. No entanto, aspectos intra-arcos, como giroversões e movimentação dentária mesiodistal ou vestibulolingual, nesta fase da dentadura, podem ser considerados normais e reversíveis, pois, até mesmo na época da esfoliação dos primeiros dentes decíduos, esses apresentam mobilidade e alterações individuais "momentâneas". Gabaritos não seriam necessários para avaliar posições dentárias individuais nem mesmo determinar formas de arcos individuais. Neste estágio de desenvolvimento da dentição, a relação do arco superior ocluindo com o inferior é que determina o diagnóstico de má oclusão.

Outros autores estudaram a forma geométrica de arcos dentários, usando pontos oclusais vestibulares, semelhantes aos aqui aplicados, para posicionar seus gabaritos ${ }^{5,8,9,10}$. No entanto, verificou-se, no presente estudo, que os pontos disto-vestibula- res das cúspides dos segundos molares decíduos referenciados para sobrepor seus gabaritos são mais linguais e estreitam o arco dentário nas extremidades. Isso leva à falsa impressão de que o segundo molar decíduo está inclinado para lingual, o que não ocorre na maioria das vezes. Resultados falsonegativos que poderiam sugerir correções desnecessárias, visto que Long ${ }^{17}$ indicou normalidade somente quando todos os pontos se sobrepuseram.

A população amostral e os parâmetros de normalidade sugeridos pelos autores que se propuseram a estudar a morfologia dos arcos dentários decíduos podem não ter se aplicado à grande variedade de formas encontradas nessa fase.

Os dados desse trabalho revelaram, independentemente do tipo de arco avaliado, superior ou inferior, resultado falso-negativo superior ao falsopositivo. Deve-se ressaltar também a sensibilidade e especificidade do gabarito, sendo que, em todos os casos, observou-se alta sensibilidade e baixa especificidade. Esse resultado demonstrou que o gabarito tem alta capacidade de identificar má oclusões em populações sabidamente alteradas e baixa capacidade de identificar oclusões normais em populações sabidamente normais. Valores falso-positivos diagnosticados com o gabarito não indicariam correções de más oclusões e valores falso-negativos sugeririam ao profissional a iniciativa de informar os pais e a população sobre a alta incidência de más oclusões e, assim, alertá-los para a necessidade de sua correção.

A fase da dentadura decídua constitui etapa transitória rápida e os dentes começam seu processo de esfoliação por volta dos 5 aos 6 anos de idade, iniciando o processo de movimentação no alvéolo. Além disso, pacientes iniciam seu desenvolvimento praticando hábitos bucais viciosos, que podem ser removidos nos primeiros anos de vida (3 a 4) e a posição dentária pode ser melhorada fisiologicamente. Pode-se considerar, então, que gabaritos analisam somente aspectos intra-arcos não determinantes nessa fase da dentadura, sendo auxiliares no diagnóstico clínico. 


\section{CONCLUSÕES}

A partir da amostra estudada e segundo a metodologia adotada, pode-se concluir que:

- os critérios clínicos que identificam uma oclusão normal ao se avaliar a dentadura decídua foram mais eficientes que os gabaritos testados, pois os gabaritos discordaram do diagnóstico clínico normal em $78 \%$ dos casos;

- dos casos estudados, 80\% que possuíam erros morfológicos pelo diagnóstico clínico concordaram com o diagnóstico pelo gabarito, houve diver- gência de resultados em 20\% dos casos;

- os resultados não foram favoráveis ao emprego clínico dos gabaritos propostos para diagnosticar a normalidade ou anormalidade da dentadura decídua.

\title{
Diagrams application in the deciduous dentition analysis
}

\begin{abstract}
Introduction: Recently, diagrams have been developed in order to analyze normality and malocclusion in the deciduous dentition. Aim: The aim of this study is to apply the diagrams developed by Long in 59 cast models of normal dentitions (group A) and 139 abnormal (group B), in order to evaluate its clinical efficiency before the known clinical criteria, which characterizes normal occlusion and malocclusion. Methods: Group A, with 118 casts of patients with normal occlusion, was stratified in 42 models of superior arches and 36 of inferior ones of type I of Baume; 17 superior casts and 23 inferior ones of arches of type II. Group B, with 278 casts with different types of malocclusions, was composed of 78 superior arches and 75 inferior ones of type 1; 61 superior arches and 64 inferior ones of type II. A total of 396 casts were examined. Results: The Kappa test between diagrams and clinical criteria demonstrated low concordance between diagnosis of normality and abnormality of the dentition, regardless of the type of arch evaluated. The diagrams disagree in $78 \%$ of the cases clinically diagnosed as normal and in $20 \%$ of those with morphological errors. Conclusion: the proposed diagrams don't apply to the primary dentition evaluation.
\end{abstract}

Key words: Deciduous dentition. Morphologic analysis. Normal occlusion. Deciduous occlusion. Malocclusion.

\section{REFERÊNCIAS}

1. ALMEIDA, M. Estudo da morfologia do arco decíduo tipo I e II de Baume, pela geometria analítica: contribuição para seu estudo. 1976. Dissertação (Mestrado)-Faculdade de Odontologia, Universidade de São Paulo, São Paulo, 1976.

2. ALMEIDA, R. R.; GARIB, D. G.; HENRIQUES, J. F. C.; ALMEIDA, M. R.; ALMEIDA, R. R. Ortodontia preventiva e interceptora: mito ou realidade? Rev. Dental Press Ortodon. Ortop. Facial, Maringá, v. 4, n. 6, p. 87-108, nov./dez. 1999.

3. BACCETTI, T.; FRANCH, L.; MCNAMARA JR., J. A.; TOLLARO, I. Early dentofacial features of Class II malocclusion. A longitudinal study from the deciduous dentition through the mixed dentition. Am. J. Orthod. Dentofacial Orthop.,
St. Louis, v. 111, no. 5, p. 502-509, May 1997.

4. BAUME, L. J. Physiological tooth migration and its significance for the development of occlusion: the biogenetic course of the deciduous dentition. J. Dent. Res., Chicago, v. 29, no. 2, p. 123-132, Apr. 1950.

5. BeGOLE, E. A.; LYEW, R. C. A new method for analyzing change in dental arch form. Am. J. Orthod. Dentofacial Orthop., St. Louis, v. 113, no. 4, p. 394-401, Apr. 1998.

6. BISHARA, S. E.; HOPPENS, B. J.; JAKOBSEN, J. R.; KOHOUT, F. J. Changes in the molar relationship between the deciduous and permanent dentitions: a longitudinal study. Am. J. Orthod., St. Louis, v. 93, p. 19-28, Jan. 1988. 
7. CARREA, J. U. Ensayos odontometricos. 1920. Tese (Doutorado)-Escuela de Odontologia de la Facultad de Ciências Médicas, Buenos Aires, 1920.

8. CURRIER, J. H. A computerized geometric analysis of human dental arch form. Am. J. Orthod., St. Louis, v. 56, no. 1/6, p. 164-179, Aug. 1969.

9. DAVIS, L. M.; BeGOLE, E. A. Evaluation of orthodontic relapse using the cubic spline function. Am. J. Orthod. Dentofacial Orthop., St. Louis, v. 113, no. 6, p. 300-306, Mar. 1998.

10. FELTON, J. M.; SINCLAIR, P. M.; JONES, D. L.; ALEXANDER, R. G. A computerized analysis of the shape and stability of mandibular arch form. Am. J. Orthod. Dentofacial Orthop. St. Louis, v. 92, p. 478-483, Dec. 1987.

11. FOSTER, T. D.; GRUNDY, M. C. Oclusal changes from primary to permanent dentitions. Br. J. Orthod., Oxford, v. 13, no. 4, p. 187-203, Oct. 1986.

12. FOSTER, T. D.; HAMILTON, M. C. Occlusion in the primary dentition. Br. Dent. J., London, v. 126, no. 2, p. 76-79, Jan. 1969.

13. GURGEL, J. A.; ALMEIDA, R. R.; DELL'ARINGA, A. R.; MARINO, V. C. C. A terapia multidisciplinar no tratamento da respiração bucal e do hábito prolongado de sucção digital ou de chupeta. Rev. Dental Press Ortodon. Ortop. Facial, Maringá, v. 8, n. 3, p. 81-91, maio/jun. 2003.

14. INTERLANDI, S. Diagrama de contorneamento ortodôntico para técnica do arco contínuo (Straight wire). Ortodontia, São Paulo, v. 35, n. 4, p. 91-105, out./dez. 2002.

15. ISSÁO, M.; GUEDES PINTO, A. C. Manual de Odontopediatria. 4th ed. São Paulo: Artes Médicas, 1978

16. JANSON, G. R. P.; SILVA, C. C. A.; HENRIQUES, J. F. C.; FREITAS, M. R.; GURGEL, J. A.; KAWAUCHI, M. Y. Correção da sobremordida com o aparelho guia de erupção. Apresentação de dois casos clínicos. Rev. Dental Press Ortodon. Ortop. Facial, Maringá, v. 3, n. 1, p. 32-46, jan./fev. 1998.

17. LONG, S. M. Análise morfológica da dentadura decídua através da sobreposição de gabaritos para arcos tipo I e II de Baume, gerados por computação eletrônica. 1999. 90 f. Dissertação (Doutorado)-Faculdade de Odontologia Universidade de São Paulo, São Paulo, 1999.

18. LONG, S. M.; CHELOTTI, A. Aplicação clínica de gabaritos para a dentadura decídua gerados por computação eletrônica: análise de Long \& Chelotti. J. Bras. Odontopediatr. Odontol. Bebê, Curitiba, v. 5, n. 24, p. 141-146, mar./abr. 2002.

19. MATHIAS, R. S. Prevalência de algumas anomalias de oclusão na dentição: mordida cruzada posterior, apinhamento anterior, mordida aberta anterior e relação terminal de segundos molares decíduos. 1984. Dissertação (Mestrado)Faculdade de Odontologia, Universidade de São Paulo, São Paulo, 1984

20. MELSEN, B.; STENSGAARD, K.; PEDERSEN, J. Sucking habits and their influence on swallowing pattern and prevalence of malocclusion. Eur. J. Orthod., Oxford, v. 1, p. 271-280, Mar. 1979.

21. MOYERS, R. E. Development of occlusion. Dent. Clin. North Am., Philadelphia, v. 13, no. 3, p. 523-536, July. 1969

22. NANDA, R. S.; KHAN, I.; ANAND, R. Effect of oral habits on the occlusion in preschool children. J. Dent. Child., Fulton, v. 39, no. 6, p. 449-452, Nov./Dec. 1972.

23. NGAN, P.; FIELDS, H. W. Orthodontic diagnosis and treatment planning in the primary dentition. ASDC J. Dent. Child. Chicago, v. 62, no. 1, p. 25-33, 1996

24. NGAN, P.; WEI, S. H. Y. Treatment of posterior crossbite in the primary and early mixed dentitions. Quintessence Int., Berlin, v. 21 , no. 6 , p. $451-459,1990$.

25. POPOVICH, F.; THOMPSON, G. W. Evaluation of preventive and interceptive orthodontic treatment between three and eighteen years of age. In: COOK, J. Transactions of the Third International Orthodontics Congress. St. Louis: C. V. Mosby, 1975.

26. SILVA FILHO, O. G.; REGO, M. V. N. N.; SILVA, P. R. B.; SILVA, F. P. L.; OZAWA, T. O. Relação intra-arco na dentadura decídua: diastemas, ausência de diastemas e apinhamento. Ortodontia, São Paulo, v. 35, n. 4, p. 8-20, out./dez. 2002.
27. SILVA FILHO, O. G.: CAPELOZZA FILHO, L.; FORNAZARI, R F.; CAVASSAN, A. O. Expansão rápida da maxila. Um ensaio sobre sua estabilidade. Rev. Dental Press Ortodon. Ortop. Facial, Maringá, v. 8, n. 1, p. 17-36, jan./fev. 2003.

28. WIESLANDER, L. Long-term effect of treatment with the headgear-Herbst appliance in the early mixed dentition. Stability or relapse? Am. J. Orthod. Dentofacial Orthop., St. Louis, v. 104, no. 4, p. 319-329, Oct. 1993.

29. ZANNET, C. G.; MENEZES, A. C. S. C.; BARBOSA, C. S.; FAVA, M.; DI NICOLO, R. Avaliação dimensional de arcos dentários decíduos utilizando os gabaritos de Long. Cienc. Odontol. Bras., São José dos Campos, v. 5, n. 3, set./dez. 2002. Disponível em: <http://www.fosjc.unesp.br/cob/artigos/ v5n3_07.pdf>. Acesso em: 22 jan. 2008.

30. ZYTKIEVITZ, E. Estudo do tamanho e forma dos arcos dentários decíduos de pré-escolares leucodermas de Curitiba. 1992. Dissertação (Mestrado)-Universidade Federal de Santa Catarina, Florianópolis, 1992.

\section{Endereço para correspondência}

Marília Inez Figueiredo

Rua Piauí - 1190/ 403 - Funcionários

CEP: 30.150-321 - Belo Horizonte / MG

E-mail: figueiredomariliainez@gmail.com 